\title{
The Association of Bovine PPARGC1A and OPN Genes with Milk Composition in Two Independent Holstein Cattle Populations
}

\author{
H. Khatib, ${ }^{* 1}$ I. Zaitoun, ${ }^{\star}$ J. Wiebelhaus-Finger, ${ }^{*}$ Y. M. Chang, † and G. J. M. Rosa* \\ *Department of Dairy Science, University of Wisconsin, Madison 53706 \\ †Genetic Epidemiology Division, St. James's University Hospital, Leeds LS9 7TF, United Kingdom
}

\begin{abstract}
Many studies have reported quantitative trait loci on chromosome 6 that affect milk production traits in dairy cattle. Osteopontin $(O P N)$ and peroxisome proliferator activated receptor gamma coactivator 1 alpha $(P P A R$ $G C 1 A$ ) are located in the middle of chromosome 6 about $6 \mathrm{Mb}$ apart, which is approximately $12 \mathrm{cM}$. The objective of this study was to investigate the association of $O P N$ and PPARGC1A variants with milk production traits in 2 independent Holstein cattle populations: the University of Wisconsin (UW) daughter design and the Cooperative Dairy DNA Repository (CDDR) granddaughter design resource populations. For OPN, 891 cows from the UW resource population were genotyped for the $\mathrm{C} / \mathrm{T}$ polymorphism reported previously in the CDDR population. Additive effects were significant for fat percentage, protein percentage, and fat yield in the UW resource population. These results are consistent with previous studies that have shown significant association of $O P N$ variants with milk composition traits. The association between PPARC1A variants was investigated in UW and CDDR resource populations using 2 single nucleotide polymorphisms. For the UW resource population, additive effects were significantly increased for protein percentage and decreased for milk yield. Dominance effects were not significant for any of the examined traits. For the CDDR population, $P P A R$ $G C 1 A$ was associated with a significant increase in milk protein percentage and in SCS. Thus, in UW and CDDR populations, PPARGC1A was associated with a significant increase in milk protein percentage in contrast to association results previously reported for the German Holstein population.
\end{abstract}

Key words: $O P N, P P A R G C 1 A$, candidate gene, single nucleotide polymorphism

Received December 4, 2006

Accepted February 4, 2007.

${ }^{1}$ Corresponding author: hkhatib@wisc.edu

\section{INTRODUCTION}

In dairy cattle and other livestock species, there is increasing interest in using a positional candidate gene approach for the identification of the actual genes that control economically important traits. At present, candidate quantitative trait genes are chosen for association studies because of previous linkage mapping studies and comparative biological functions in the same or other species (Rothschild and Soller, 1997). Many studies have reported QTL on multiple chromosomes that affect milk production and health traits in dairy cattle. Chromosome 6 contains the highest number of reported QTL (Khatkar et al., 2004). Meta-analysis of these QTL studies suggests that one QTL affecting milk, fat, and protein yield and fat and protein percentages is located around $50 \mathrm{cM}$, near microsatellite BM143 (Khatkar et al., 2004). There was also evidence for a second QTL affecting milk yield and protein percentage around $87 \mathrm{cM}$. For the QTL in the middle of the chromosome, 8 studies reported QTL affecting milk yield, 11 reported QTL affecting protein percentage, 5 reported QTL affecting protein yield, and 7 reported QTL affecting fat percentage (Khatkar et al., 2004).

Ron et al. (2001) localized a QTL affecting protein percentage to a confidence interval of $4 \mathrm{cM}$ in the region near $50 \mathrm{cM}$ on chromosome 6 . Recently, Olsen et al. (2005) positioned a QTL affecting milk production traits at an interval of $420 \mathrm{~kb}$ between the genes ABCG2 and $L A P 3$ on bovine chromosome 6 . This narrow region harbors only 6 genes including the osteopontin (OPN) gene. Based on the aforementioned QTL studies, several groups investigated possible associations between genes in this region and milk production traits in dairy cattle. Weikard et al. (2005) reported significant association between a single nucleotide polymorphism (SNP) in intron 9 of the peroxisome proliferator activated receptor gamma coactivator 1 alpha (PPARGC1A) gene (close to microsatellite BM143) and milk fat yield in the German Holstein population. Leonard et al. (2005) and Schnabel et al. (2005) reported an association between $O P N$ and milk protein percentage in the North American Holstein population. Furthermore, Schnabel 
et al. (2005) presented several lines of evidence for a quantitative trait nucleotide, located upstream of $O P N$ promoter region, causing variation in milk protein percentage. On the other hand, Cohen-Zinder et al. (2005) reported the identification of a causative mutation in ABCG2 affecting milk yield and composition and excluded the $O P N$ mutation reported in the Schnabel study from causality. The results for these conflicting candidate genes for the QTL in the middle of chromosome 6 necessitate additional populations to be tested for these genes (de Koning, 2006). In this study, the association of $O P N$ variants with milk composition was investigated in an independent Holstein population for the validation of results previously obtained in a different granddaughter design Holstein population. In addition, the association between PPARGC1A variants, also located in the middle of chromosome 6 , and milk production traits was also investigated in the University of Wisconsin (UW) daughter design and in the Cooperative Dairy DNA Repository (CDDR) granddaughter design resource populations. The OPN and PPARGC1A are about $6 \mathrm{Mb}$ apart, which is about $12 \mathrm{cM}$ for this region of chromosome 6 (http://www.ncbi.nlm.nih.gov/ mapview).

\section{MATERIALS AND METHODS}

\section{Populations and Data}

Blood samples ( $\mathrm{n}=931$ ) were obtained from the UW resource population. This population was originally created to search for QTL in association with susceptibility to paratuberculosis (Gonda et al., 2006). Cows were sampled from 300 herds scattered throughout the United States. The 12 sire families of this population were chosen because they included large numbers of daughters in production and because of relatively low pedigree relationships among the bulls. Of 66 possible pairings of sires, genetic relationships were 0.25 for 1 pair of sires, 0.125 for 7 pairs, 0.0625 for 13 pairs, and lower for the remaining 45 pairs (Gonda et al., 2006).

In addition, semen samples from 12 Holstein sires and their 581 sons were obtained from the CDDR, which is maintained by the USDA Bovine Functional Genomics Laboratory (Beltsville, MD). Predicted transmitting ability data for sons in the CDDR and yield deviation for daughters in the UW resource populations were obtained for milk, protein, and fat yields (kg), protein and fat percentages, and SCS from the USDA Animal Improvement Programs Laboratory (Beltsville, MD).

\section{OPN and PPARGC1A SNP}

It was previously shown that a $\mathrm{C} / \mathrm{T}$ polymorphism at position 8514 of $O P N$ (GenBank accession number
NW_255516) is associated with milk protein and fat percentages in 1,362 bulls obtained from 28 sire families from the CDDR population (Leonard et al., 2005). To validate this association, 891 cows from the UW resource population were genotyped for this polymorphism. The association of PPARGC1A SNP with milk production was investigated in the UW and the CDDR resource populations using 2 SNP reported by Weikard et al. (2005); SNP C/T at position 1892 and SNP A/C at position 3359. The SNP 1892 was genotyped only for the UW resource population; SNP 3359 was genotyped in both populations.

\section{SNP Genotyping}

Genomic DNA was extracted from blood samples using GFX Genomic Blood DNA Purification Kit (Amersham Biosciences, Piscataway, NJ) and from semen samples by standard methods using proteinase $\mathrm{K}$ and phenol/chloroform. Primer sequences and amplification conditions for $O P N$ were described in Leonard et al. (2005). Primers 1892F 5'-CATAGCCGGCGCCCCAGG TAAGATGCACGTTGGC-3' and 1892R 5'-CTGGTACT CCTCGTAGCTGTC-3' were used to amplify $195 \mathrm{bp}$ in intron 9 to genotype SNP C/T at position 1892 of $P P A R$ GC1A. Primers 3359F 5'-GCGAGCACGGTGTTACAT TACTAAGGAGAGTTGGCTAG-3' and 3359R 5'-GTT GTGTTGCACTCAATGGAC-3' were used to amplify $357 \mathrm{bp}$ in the $3^{\prime}$ untranslated region ( $3^{\prime} \mathrm{UTR}$ ) of PPARGC1A to genotype SNP A/C at position 3359. Primers 1892R and 3359R were used by Weikard et al. (2005). Amplification was performed in a $15-\mu \mathrm{L}$ reaction volume, which included $25 \mathrm{ng}$ of genomic DNA, $25 \mathrm{ng}$ of each primer, $100 \mu M$ of each dNTP, $1.5 \mu \mathrm{L}$ of $10 \times$ PCR buffer (Promega, Madison, WI), and 0.2 U of Taq DNA polymerase (Promega). The temperature cycles were as follows: $95^{\circ} \mathrm{C}$ for $5 \mathrm{~min}$, followed by 32 cycles of $94^{\circ} \mathrm{C}$ for $45 \mathrm{~s}, 50^{\circ} \mathrm{C}$ for $45 \mathrm{~s}, 72^{\circ} \mathrm{C}$ for $45 \mathrm{~s}$, and a final extension at $72^{\circ} \mathrm{C}$ for $7 \mathrm{~min}$. For genotyping of SNP C/T at position 1892, PCR products were digested with the restriction enzyme HaeIII that distinguishes alleles $\mathrm{C}$ and $\mathrm{T}$ of the SNP. For genotyping of SNP A/C at position 3359, PCR products were digested with the restriction enzyme NheI that distinguishes alleles A and C. The digestion products were electrophoresed on a $2.5 \%$ agarose gel.

\section{Statistical Analysis}

The association of $O P N$ and PPARGC1A SNP with production traits was studied in 2 Holstein populations (CDDR and UW). The CDDR phenotypic data referred to bulls' PTA data for milk, protein and fat yield, protein and fat percentages, and SCS. The UW resource population data was composed of yield deviations of cows for the same traits. 
CDDR Population. Associations between $O P N$ variants and the phenotypic traits recorded in the CDDR population were published by Leonard et al. (2005). Therefore, in the present work only the PPARGC1A locus was considered in this population. Because the CDDR data are PTA, effects of an additional positive allele in the sons of a sire are necessarily additive. Hence, for this data set an allele substitution model having the following structure was used for the analysis of each trait:

$$
y_{i j}=\mu+s_{i}+\beta x_{i j}+\varepsilon_{i j}
$$

where $y_{i j}$ is the PTA value relative to son $j$ of sire $i, \mu$ is a general constant (intercept), $s_{i}$ is the fixed effect of sire $i, \beta$ is the regression coefficient representing half of the allele substitution effect $(\alpha / 2), x_{i j}$ is the number of A alleles $(0,1$, or 2$)$ at the PPARGC1A locus of son $j$ of sire $i$, and $\varepsilon_{i j}$ is a residual term. Reliabilities of the sons' PTA were incorporated as weights in the model to obtain weighted least squares estimates of the allele substitution effects.

UW Resource Population. Because the UW resource data are individual cow records, allele effects are not necessarily additive. Hence, data relative to each trait in the UW resource population were analyzed using the following fixed effects model:

$$
y_{i j k l}=\mu+s_{i}+d_{i j} \tau+o_{k}+p_{l}+\varepsilon_{i j k l},
$$

where $y_{i j k l}$ represents the yield deviation of daughter $j$ of sire $i ; \tau$ is an effect associated with $M$. paratuberculosis infection status; $d_{i j}$ is an indicator variable assuming the values 0 and 1 for noninfected and infected cows, respectively; $o_{k}$ is the effect of the OPN genotype (k = $\mathrm{CC}, \mathrm{CT}$, or TT); and $p_{l}$ is the effect of the PPARGC1A genotype ( $1=\mathrm{AA}, \mathrm{AC}$, or $\mathrm{CC})$. The remaining terms in the model are as previously defined. Both $O P N$ and PPARGC1A were analyzed in the same model to control for variation introduced by each locus. All interaction terms were included in the error term. This was not done for CDDR data because only the PPARGC1A locus was considered in this population at this time. Additive genetic effect of each locus was estimated as half of the difference between the 2 homozygous groups, as $\left(\hat{o}_{C C}-\right.$ $\left.\hat{o}_{T T}\right) / 2$ for $O P N$ and $\left(\hat{p}_{A A}-\hat{p}_{C C}\right) / 2$ for PPARGC1A. The dominance effect was estimated as the difference between the heterozygous group and the average of the 2 homozygous groups in each locus. The analyses were implemented using the GLM procedure of SAS (SAS Institute, 1999).
Table 1. Estimates $( \pm \mathrm{SE})$ of additive and dominance effects associated with the single nucleotide polymorphism at position 8514 of the osteopontin $(O P N)$ gene in the University of Wisconsin resource population

\begin{tabular}{lcc}
\hline Trait & \multicolumn{1}{c}{ Additive effect } & Dominance effect \\
\hline Fat yield & $9.32( \pm 3.79)^{*}$ & $4.23( \pm 4.55)$ \\
Fat percentage & $0.0496( \pm 0.0133)^{* * *}$ & $-0.0020( \pm 0.0160)$ \\
Milk yield & $-83.63( \pm 101.6)$ & $133.0( \pm 122.0)$ \\
Protein yield & $3.75( \pm 2.70)$ & $4.79( \pm 3.24)$ \\
Protein percentage & $0.0251( \pm 0.0062)^{* * *}$ & $0.0036( \pm 0.0074)$ \\
SCS & $0.0085( \pm 0.0583)$ & $0.0100( \pm 0.0698)$ \\
\hline
\end{tabular}

$* P<0.05 ; * * * P<0.001$.

\section{RESULTS}

\section{OPN}

Frequencies of CC, CT, and TT genotypes in the UW population were $0.23,0.51$, and 0.26 , respectively, and were in close accord with Hardy-Weinberg (H-W) expectations. Table 1 shows the estimates of the additive and dominance genotypic effects SNP C/T at position 8514 of $O P N$ for milk yield and composition traits and for SCS in the UW resource population. Additive effects were significant for fat percentage $(P<0.0001)$, protein percentage $(P<0.0001)$, and fat yield $(P=0.014)$. Dominance effects were not significant for any of the examined traits.

\section{PPARGC1A}

The SNP C/T at position 1892 was genotyped only for the UW resource population, and the frequencies of genotypes CC, CT, and TT of SNP 1892 were 33.1, 65.0, and $1.9 \%$, respectively. Genotype frequencies differed strongly and with high significance from $\mathrm{H}-\mathrm{W}$ expectations. In particular, there was a marked deficiency of TT homozygotes, raising the possibility of a recessive semilethal associated with this genotype. Association tests between SNP 1892 and milk production traits in the UW resource population were not significant for any of the examined traits (data not shown). Therefore, this SNP was not tested further in the CDDR population. In contrast, the association between SNP A/C at position 3359 and milk production traits was examined in UW and CDDR populations. For the UW population, the frequencies of genotypes $\mathrm{AA}, \mathrm{AC}$, and $\mathrm{CC}$ at this SNP were $16.3,50.6$, and $33.1 \%$, respectively, and for the CDDR population, the frequencies of these genotypes were $12.3,43.0$, and $44.7 \%$, respectively. In both populations genotype frequencies were in close accord with $\mathrm{H}-\mathrm{W}$ expectations. Table 2 shows estimates of additive and dominance effects of PPARGC1A locus in the UW population and the average allele substitution effects for production traits in the CDDR population. 
Table 2. Estimates $( \pm \mathrm{SE})$ of additive and dominance effects associated with the single nucleotide polymorphism at location 3359 of the peroxisome proliferator-activated receptor gamma coactivator 1 alpha (PPAR$G C 1 A)$ gene in the University of Wisconsin (UW) resource population and estimates $( \pm \mathrm{SE}$ ) of the average allele substitution effect (allele A) in the $\mathrm{CDDR}^{1}$ population

\begin{tabular}{|c|c|c|c|}
\hline Trait & Additive effect ${ }^{2}$ & Dominance effect ${ }^{2}$ & Allele substitution ${ }^{3}$ \\
\hline Fat yield & $-4.18( \pm 3.85)$ & $5.40( \pm 4.75)$ & $-1.20( \pm 2.82)$ \\
\hline Fat percentage & $0.0189( \pm 0.0138)$ & $0.0011( \pm 0.0171)$ & $0.008( \pm 0.010)$ \\
\hline Milk yield & $-238.6( \pm 102.6)^{*}$ & $128.2( \pm 126.6)$ & $-97.6( \pm 73.0)$ \\
\hline Protein yield & $-3.04( \pm 2.73)$ & $6.58( \pm 3.37)$ & $0.264( \pm 1.908)$ \\
\hline Protein percentage & $0.0185( \pm 0.0064)^{* *}$ & $0.0107( \pm 0.0080)$ & $0.012( \pm 0.004)^{*}$ \\
\hline SCS & $-0.0003( \pm 0.0586)$ & $0.0206( \pm 0.0724)$ & $0.042( \pm 0.020)^{*}$ \\
\hline
\end{tabular}

${ }^{1}$ Cooperative Dairy DNA Repository.

${ }^{2}$ Estimates from yield deviations from the UW resource population.

${ }^{3}$ Estimates from PTA from the CDDR population.

$* P<0.05 ; * * P<0.01$.

Significant effects were found for milk yield in the UW population $(P=0.020)$; for protein percentage in UW and CDDR populations $(P=0.004$ and 0.016 , respectively); and for SCS in the CDDR population $(P=$ 0.038). Dominance effects, which were included in the model for analysis of the UW data, were not significant for any of the examined traits. Direction of additive effect (UW) and allele substitution effect (CDDR) were the same for all traits, except protein yield and SCS. In particular, the A allele was associated with significant positive effects on protein percentage in both populations. Also, there was an indication of a decrease in milk yield for this allele in both populations, although it was not significant in the CDDR population. This is not surprising given that the correlation between protein percentage and milk yield in the CDDR population was -0.40 (Khatib et al., 2005).

\section{DISCUSSION}

The results of the present study are consistent with previous studies that have shown significant association of $O P N$ variants with milk composition traits (Cohen et al., 2004; Leonard et al., 2005; Schnabel et al., 2005). Cohen et al. (2004) investigated the association of $12 \mathrm{SNP}$ in 10 genes, located in the region of the microsatellite BM143 in the middle of chromosome 6, and milk composition in 420 Holstein bulls. Among these genes, $O P N$ showed the highest linkage disequilibrium effects on protein percentage. In addition, based on expression analysis in mammary gland in cattle (Cohen et al., 2004) and on abnormal differentiation of mammary gland in antisense transgenic $O P N$ mice (Nemir et al., 2000), Cohen et al. (2004) concluded that $O P N$ was the key candidate gene affecting milk protein percentage. Conversely, in a subsequent study, CohenZinder et al. (2005) sequenced bulls with known QTL for SNP OPN3907, reported by Schnabel et al. (2005) to be the quantitative trait nucleotide, and found that this SNP region was hyper variable and that 17 of 18 bulls were heterozygous for at least one polymorphism. Accordingly, they concluded that $A B C G 2$ but not $O P N$ is the quantitative trait gene affecting milk composition in the middle of chromosome 6 (Cohen-Zinder et al., 2005).

The PPARGC1A gene was chosen for association test with milk fat traits because of several QTL studies that showed effects of PPARGC1A region on milk production and because of possible involvement of this gene in fat metabolism (Weikard et al., 2005). The authors reported a significant association between SNP 1892 in intron 9 and milk yield and milk fat traits in 434 bulls from the German Holstein population. The SNP 3359 in the $3^{\prime}$ UTR showed some trend of association with fat traits, but it did not reach the significance level of $5 \%$, so the authors attributed this association to the linkage disequilibrium between the 2 SNP.

In contrast to Weikard et al. (2005), in the current study a significant association was found between SNP 3359 and protein percentage and milk yield, whereas SNP 1892 did not show significant association with any of the examined traits. These conflicting findings between results from this study and results from Weikard et al. (2005) could be explained by the 2 different genetic backgrounds of the studied populations or by the different number of samples included in each study. In the current study, PPARGC1A was genotyped in 2 independent populations with more than 1,400 individuals, whereas in the study by Weikard et al. (2005) 434 bulls were genotyped. Also, different associations of different SNP in the same gene in 2 or more populations could be a result of linkage disequilibrium of these SNP with the causative mutation in the same gene or in other genes. The SNP showing significant association with milk production traits would provide an excellent opportunity for marker-assisted selection (MAS) programs in dairy cattle. The aim of MAS is to substitute selection at the DNA level for selection on the basis of 
phenotype (Soller, 1994). Also, MAS can increase allele frequencies of the favorable alleles, as has been shown for the calpain and calpastatin genes in beef cattle (Pollak, 2005). However, for MAS to be efficient in genetic improvement programs, it is assumed that the effects of genes used in MAS are known and are consistent in different genetic backgrounds and environments (Dekkers and Hospital, 2002). The inconsistent association results between our data and Weikard's necessitate further investigation prior to applications in MAS programs.

\section{ACKNOWLEDGMENTS}

This research was supported by start-up funding from the University of Wisconsin-Madison and by The Draper Technology Innovation Fund from Graduate School, the University of Wisconsin-Madison. We thank George E. Shook for providing blood samples from the UW resource population. We thank the staff and administration of the USDA Bovine Functional Genomics Laboratory for providing semen samples from the CDDR population.

\section{REFERENCES}

Cohen, M., E. Seroussi, M. R. Band, H. A. Lewin, J. K. Drackley, D. M. Larkin, A. Everts-van der Wind, J. Heon-Lee, J. J. Loor, M. Shani, J. I. Weller, and M. Ron. 2004. SPP1 is a candidate gene for the QTL affecting milk protein concentration on BTA6 in Israeli Holstein. 29th Int. Soc. Anim. Genetics, Tokyo, Japan.

Cohen-Zinder, M., E. Seroussi, D. M. Larkin, J. J. Loor, A. Evertsvan der Wind, J. H. Lee, J. K. Drackley, M. R. Band, A. G. Hernandez, M. Shani, H. A. Lewin, J. I. Weller, and M. Ron. 2005. Identification of a missense mutation in the bovine ABCG2 gene with a major effect on the QTL on chromosome 6 affecting milk yield and composition in Holstein cattle. Genome Res. 15:936-944.

Dekkers, J. C. M., and F. Hospital. 2002. The use of molecular genetics in the improvement of agricultural populations. Nat. Rev. Genet. $3: 22-32$. de Koning, D. J. 2006. Conflicting candidates for cattle QTLs. Trends Genet. 22:301-305.

Gonda, M. G., Y. M. Chang, G. E. Shook, M. T. Collins, and B. W. Kirkpatrick. 2006. Genetic variation of Mycobacterium avium ssp. paratuberculosis infection in US Holsteins. J. Dairy Sci. 89:1804-1812.

Khatib, H., E. Heifetz, and J. C. Dekkers. 2005. Association of the protease inhibitor gene with production traits in Holstein dairy cattle. J. Dairy Sci. 88:1208-1213.

Khatkar, M. S., P. C. Thomson, I. Tammen, and H. W. Raadsma. 2004. Quantitative trait loci mapping in dairy cattle: Review and meta-analysis. Genet. Sel. Evol. 36:163-190.

Leonard, S., H. Khatib, V. Schutzkus, Y. M. Chang, and C. Maltecca. 2005. Effects of the osteopontin gene variants on milk production traits in dairy cattle. J. Dairy Sci. 88:4083-4086.

Nemir, M., D. Bhattacharyya, X. Li, K. Singh, A. B. Mukherjee, and B. B. Mukherjee. 2000. Targeted inhibition of osteopontin expression in the mammary gland causes abnormal morphogenesis and lactation deficiency. J. Biol. Chem. 275:969-976.

Olsen, H. G., S. Lien, M. Gautier, H. Nilsen, A. Roseth, P. R. Berg, K. K. Sundsaasen, M. Svendsen, and T. H. Meuwissen. 2005. Mapping of a milk production quantitative trait locus to a 420 $\mathrm{kb}$ region on bovine chromosome 6. Genetics 169:275-283.

Pollak, E. J. 2005. Application and impact of new genetic technologies on beef cattle breeding: a 'real world' perspective. Aust. J. Exp. Agric. 45:739-748.

Ron, M., D. Kliger, E. Feldmesser, E. Seroussi, E. Ezra, and J. I. Weller. 2001. Multiple quantitative trait locus analysis of bovine chromosome 6 in the Israeli Holstein population by a daughter design. Genetics 159:727-735.

Rothschild, M., and M. Soller. 1997. Candidate gene analysis to detect genes controlling traits of economic importance in domestic livestock. Probe 8:13-20.

SAS Institute. 1999. SAS OnlineDoc, Version 8. SAS Institute Inc., Cary, NC.

Schnabel, R. D., J. J. Kim, M. S. Ashwell, T. S. Sonstegard, C. P. Van Tassell, E. E. Connor, and J. F. Taylor. 2005. Fine-mapping milk production quantitative trait loci on BTA6: Analysis of the bovine osteopontin gene. Proc. Natl. Acad. Sci. USA 102:68966901.

Soller, M. 1994. Marker assisted selection-An overview. Anim. Biotechnol. 3:193-201.

Weikard, R., C. Kuhn, T. Goldammer, G. Freyer, and M. Schwerin. 2005. The bovine PPARGC1A gene: Molecular characterization and association of an SNP with variation of milk fat synthesis. Physiol. Genomics 21:1-13. 